\title{
Performance Analysis of Independent Component Analysis Algorithms for Multi-user Detection of DS-CDMA
}

\author{
G. Thavasi Raja , P. Krishna Chaitanya and R. Malmathanraj \\ Department of Electronics and Communication Engineering \\ National Institute of Technology (NIT), Tiruchirappalli \\ Tamil Nadu- 620 015, India
}

\begin{abstract}
The conventional detection process of direct sequence code division multiple access (DS-CDMA) is limited by multiple access interference (MAI) due to loss of orthogonality between spreading waveforms in multipath propagation channel environment.. In this paper RADICAL Independent Component Analysis (ICA) algorithm is proposed for detection of DS-CDMA signals and compared with FastICA, JADE ICA algorithm. Independent component analysis (ICA) is statistical technique based on higher order statistics, represent set random variables as linear transformation of statistically independent components and these conditions are satisfied in multi-user CDMA environment. Conventional methods mitigate sources of interference by taking into account all available information and at times seeking additional information channel characteristics, direction of arrival, etc. Combining an ICA element to conventional signal detection reduces multiple access interference (MAI) and enables a robust, computationally efficient structure. The proposed algorithm takes advantage of differential entropy estimation and converges quickly. Bit error rate simulations of these algorithms have been given and compared for different number of users, SNR. The simulation results show that RADICAL ICA algorithm performs best on detecting the source signals from the mixed CDMA signals.
\end{abstract}

Keywords ICA, DS-CDMA, MAI, RADICAL, JADE

\section{INTRODUCTION}

The main objective of communication is reliable transfer of information between two parties, in the sense that the information reaches the intended party with as few errors as possible. Code division multiple access is emerging as the popular multiple access scheme, mainly due to its soft multiple access characteristic, robustness against fading and anti-interference capability. The main sources of errors at the detector are due to the multiple access interference (MAI), the inter symbol interference (ISI), asynchronous behavior of users and near far problem. MAI constitutes a significant bottleneck in achieving the high capacity of a direct sequence code division multiple access (DS-CDMA) system. The conventional single-user detection methods consider MAI as external noise. An alternative approach is to study and exploit the structure of MAI to achieve interference suppression. The structure of MAI has been exploited in the paper on optimum multi user detection and its sub optimal counter parts [1]. It is observed that these detectors either require complete knowledge of the MAI, training data or involve long decoding delays $[1,2]$. To overcome these limitations, a class of spectrally efficient blind detectors was proposed. However, most of the blind detection techniques in wireless communication literature [4] utilize only the second order statistics (SOS) of the received data.Independent Component Analysis (ICA) is a statistical technique based on higher order statistics (HOS), where the goal is to represent a set of random variables as a linear transformation of statistically independent components [3]. ICA based techniques are based on the assumption of non-gaussianity and independence of the sources. Fast-ICA algorithm applied for detection of DSCDMA in [5]. But the convergence is not sure in Fast-ICA process. The RAKE-ICA proposed by [6]. This method needs the information of multi-path delay time of the desired user, which is difficult to estimate. In [7] ICA has been applied using SAND algorithm. But it requires Wavelet denoising analysis to suppress the adverse effects of noise on the SAND algorithm. In this paper FastICA, JADE and RADICAL ICA algorithms applied for DS-CDMA detection. This method is very useful in a multi user CDMA environment where prior information about the user's code is generally available with the receiver. This approach can be considered as a blind approach as though the spreading codes of all the users are assumed known, no estimation of the channel impulse response is necessary to perform detection of user's signals. For simplicity, assume the CDMA model as a synchronous one where no ISI is present. Simulations have been carried out to observe variation in bit error rate as a function of signal to noise ratio and number of users.

The rest of this paper is organized as follows. Section 2 gives a description of DS-CDMA signal model. Section 3 discusses brief introduction about Independent Component Analysis, signal model of ICA and required preprocessing steps for ICA. Section 4 discusses the detection scheme using for different ICA algorithms. The simulation results discussion and concluding remarks are given in Section 5 and Section 6 respectively.

\section{SIGNAL MODEL OF DS-CDMA}

The received signal has the form,

$$
\mathrm{y}(\mathrm{t})=\sum_{\mathrm{m}=0}^{\mathrm{M}-1} \sum_{\mathrm{k}=1}^{\mathrm{K}} \mathrm{b}_{\mathrm{km}} \mathrm{a}_{\mathrm{km}} \mathrm{s}_{\mathrm{k}}(\mathrm{t}-\mathrm{mT})+\mathrm{n}(\mathrm{t})
$$

where $b_{k m}$ is $m^{\text {th }}$ symbol of the $\mathrm{k}^{\text {th }}$ user, $a_{k m}$ is attenuation factor of the $\mathrm{m}^{\text {th }}$ symbol, which may vary from symbol to symbol, $\mathrm{M}$ is number of symbols per user, $\mathrm{K}$ is number of users, $\mathrm{T}$ is the symbol duration, $\mathrm{n}(\mathrm{t})$ denotes the additive white 
Gaussian noise with zero mean and unit variance noise, and the chip sequence length (i.e., processing gain) is $C=T / T_{c}$, where $T_{c}$ is chip duration. For simplicity, from now it is assumed that $T_{c}=1$. Since the chip sequence $s_{k}($.$) is now$ continuous by definition, it includes not only the binary chips $\mathrm{s}_{\mathrm{k}}[\mathrm{i}]$, but also a chip waveform $\mathrm{p}(\mathrm{t})$. More precisely,

$s_{k}(t)=\sum_{i=0}^{C-1} s_{k}[i] P\left(t-i T_{c}\right)$

where $\mathrm{P}(\mathrm{t})$ is supported by $[0, \mathrm{Tc}]$ only. This paper assumes rectangular waveforms for each user, and hence,

$\mathrm{P}(\mathrm{t})=1$, when $0<\mathrm{t}<\mathrm{Tc}$,
$\quad=0$, otherwise

\section{ICA SIGNAL MODEL}

Independent Component Analysis (ICA) [8] involves the task of computing the matrix projection of a set of components onto another set of so called independent component. Here, the objective is to maximize the statistical independence of the outputs. If the inputs to the ICA are known to be linear instantaneous mixture of a set of sources, the ICA process provides an estimate of the original sources. Here, and in the context of this paper, neither the original sources nor the mixture matrix are known. This is the Blind Separation of Sources (BSS) [8] where the aim is to obtain a non-observable set of signals, the so-called sources, from another set of observable signals regarded as mixtures. The BSS problem can be easily tackled by exploiting the higher order signal statistics and optimization techniques. The original source vector $\mathrm{S}$ is of size $\mathrm{M} \times \mathrm{N}$ and the mixing matrix $\mathrm{A}$ is of size $\mathrm{M}$ $\mathrm{x} M$, where $\mathrm{M}$ is the number of statistical independent sources and $\mathrm{N}$ is the number of samples in each source.

The result of the separation process is the demixing matrix $\mathrm{W}$ which can be used to obtain the estimated statistical independent sources, $\hat{\mathrm{S}}$ from the mixtures. This process is described by equation (4) and a schematic illustration of the mathematical model in shown in Figure 1.

$$
\mathbf{X}=\mathbf{A S} \rightarrow \hat{\mathbf{S}}=\mathbf{W X}
$$

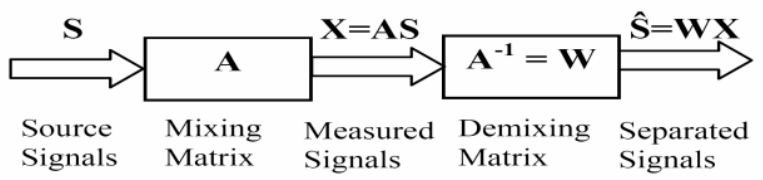

Fig 1: Schematic illustration of the mathematical model used to perform ICA decomposition

\subsection{Preprocessing for ICA}

Some preprocessing is useful before attempting to estimate $\mathrm{W}$. The observed signals should be centered by subtracting their mean value $\mathrm{E}\{\mathrm{X}\}$. Then they are whitened, which means they are linearly transformed so that the components are uncorrelated and has unit variance. Whitening can be performed via eigenvalues decomposition of the covariance matrix, $\mathrm{V} \Lambda \mathrm{V}^{\mathrm{T}}, \mathrm{V}$ is the matrix of orthogonal eigenvectors and $\Lambda$ is a diagonal matrix with the corresponding eigenvalues. The whitening is done by multiplication with the transformation matrix.

\section{DETECTION SCHEME}

The received DS-CDMA signal $\mathrm{y}$, which is combination of various independent sources, is preprocessed and applied to ICA and the sources are separated by ICA and the detection scheme is shown in the Figure 2. Various independent components are identified using PN codes and then conventional PSK detection process is done. The sources are separated by ICA and identification of the sources requires additional information. After identification, conventional detection sufficiently recovers the users' signals $\hat{b}_{k}$ from the $\hat{y}_{k}$, is received CDMA signal. Various ICA algorithms are available for BSS. Efficient algorithms are considered for the detection which is shown in Figure 3.

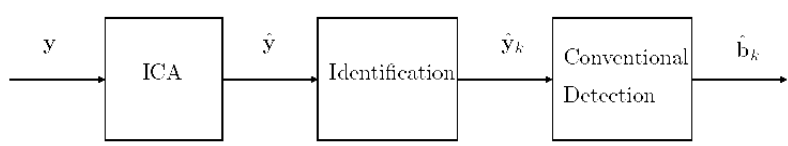

Fig 2: Detection scheme with ICA.

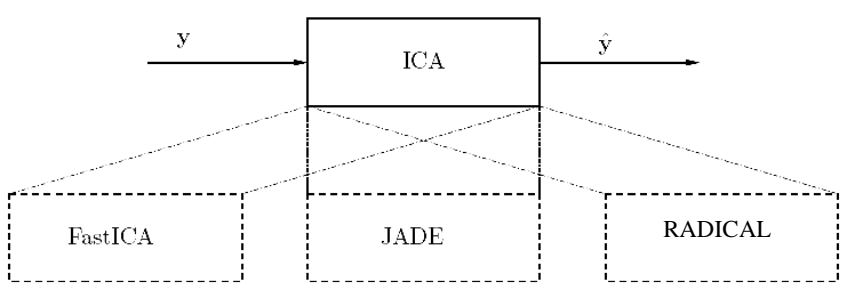

Fig 3: Choice of various ICA algorithms.

\subsection{FastICA Algorithm}

The fast algorithm for Independent Component Analysis (FastICA) proposed [12, 13,14], which can be used for blind source separation and feature extraction. The algorithm finds one at a time, all non-Gaussian independent components, regardless of their probability distribution and FastICA learning rule finds a direction, i.e. a unit vector $w$ such that the projection $\mathbf{w}^{\mathbf{T}} \mathbf{x}$ maximizes nongaussianity.

\subsection{JADE ICA Algorithm}

The next signal source separation technique is considered for analysis is the Joint Approximation Diagonalisation of Eigen matrices (JADE) algorithm [8]. This exploits the fourth order moments in order to separate the source signals from mixed signals. The operation of JADE [8] includes optimization of orthogonal contrast by finding the rotation matrix $R$ such that the cumulant matrices are as diagonal as possible, that is, given the equation (5) and components are estimated using whitening matrix and rotation matrix.

$$
R=\arg \min _{R} \sum_{i} o f f\left(R^{T} \hat{Q}_{i}^{Z} R\right)
$$

\subsection{Proposed RADICAL ICA Algorithm Based Detection}

The RADICAL (Robust, Accurate, Direct Independent Component Analysis Algorithm) [9] estimates the independent sources using differential entropy estimator based on ' $\mathrm{m}$ '-spacing estimator. The contrast function in (6) is to be minimized by RADICAL is almost equivalent to Vasicek estimator [11],

$$
\hat{H}_{\text {RADICAL }}\left(Z^{1}, \ldots \ldots, Z^{N}\right) \equiv \frac{1}{N-m} \sum_{i=1}^{N-m} \log \left(\frac{N+1}{m}\left(Z^{(i+m)}-Z^{i}\right)\right)
$$

The data vectors $\mathrm{X}_{1}, \mathrm{X} 2 \ldots \mathrm{XM}$ are assumed to be whitened using the whitening matrix P. Let $m$ be the size of spacing. 
The value of ' $\mathrm{m}$ ' is taken as $\sqrt{N}$ where $\mathrm{N}$ is the number of samples in each source. Let $\mathfrak{R}$ be the number of replicated points per original data point to eliminate the local minima problem. Straightforward principles underlie in RADICAL algorithm [10]:

1. Directly optimizes a measure of statistical independence, rather than a substitute for this measure.

2. It avoids explicit estimation of probability densities as an intermediate step.

3. Since the objective function involves one-dimensional entropy estimation it employs a well known rapidly converging and computationally efficient estimator of entropy which is robust to outliers. Higher dimensional algorithms and computational complexity has been given in [10] and concluded that RADICAL is highly robust to outliers and proved that, having efficient computational complexity of order $\mathrm{O}(\mathrm{N} \log \mathrm{N})[10]$.

\section{SIMULATION RESULTS AND DISCUSSIONS}

Simulations are done in MATLAB and DS-CDMA with BPSK modulation is considered for simulation. The ICA algorithms FastICA, JADE and RADICAL combined to conventional detector and BER simulations are done for various number of users. Also simulations are done for various values of SNR and the results are compared for low and high SNR. Figure 4 shows the simulation results of BER vs number of users of JADE, FastICA and RADICAL ICA algorithms for detection of DS-CDMA when $\mathrm{SNR}=3 \mathrm{~dB}$ and also these results are compared to the results when ICA not used. It can be observed that RADICAL and JADE algorithms are having lower BER compared to FastICA algorithm. Moreover FastICA also having low BER compared to without any ICA results. Also we can observe that as the number of users increases all ICA algorithms are giving same BER and they are meeting BER values of the results without ICA. For high number of users ICA may not give good results. If it is not giving good results ICA is overhead. To remove this ambiguity we have to use pre or post switch, but this is not the intention of this work.

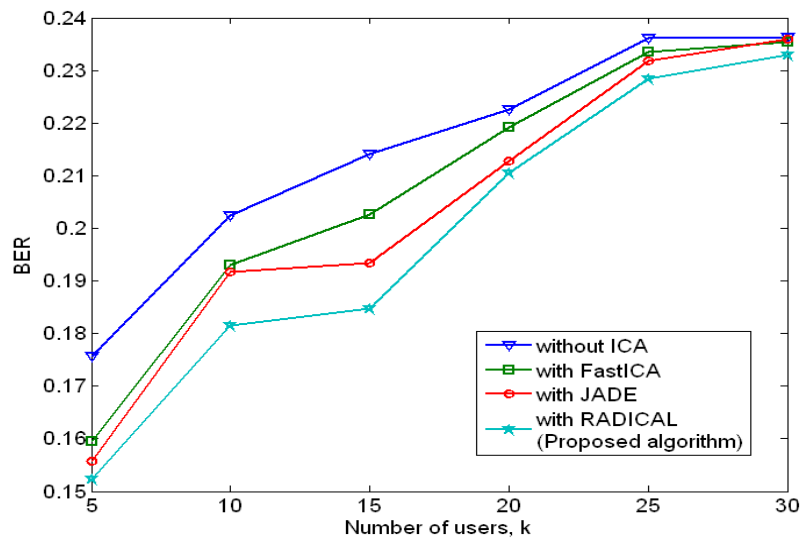

Fig 4: Comparison of ICA algorithms for detection of DS-CDMA signals in AWGN channel $(\mathrm{SNR}=3 \mathrm{~dB})$

In Figure 5, the similar comparison for $\mathrm{SNR}=4 \mathrm{~dB}$ are given that and the observations have noted as previous graph for low number of users. For high number of users ICA is still getting good results than of without ICA. From this observation, SNR also affects the results i.e. for high SNR, ICA giving good results even for high number of users. The SNR vs. BER curves for number of users $\mathrm{k}=10,20$ is given in Figure 6, Figure 7 respectively. As SNR is increased, ICA is performing well and for higher number of users high SNR is suggested. The BER performance simulations show that RADICAL ICA algorithm is the best choice for detection of DS-CDMA and also it is computationally efficient [10] than other ICA algorithms.

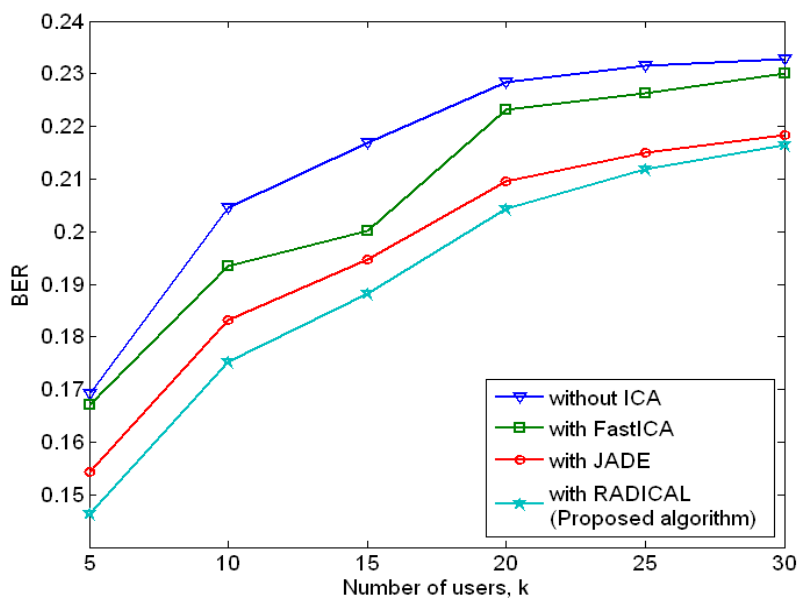

Fig 5: Comparison of ICA algorithms for detection of DS-CDMA signals in AWGN channel (SNR = 4dB)

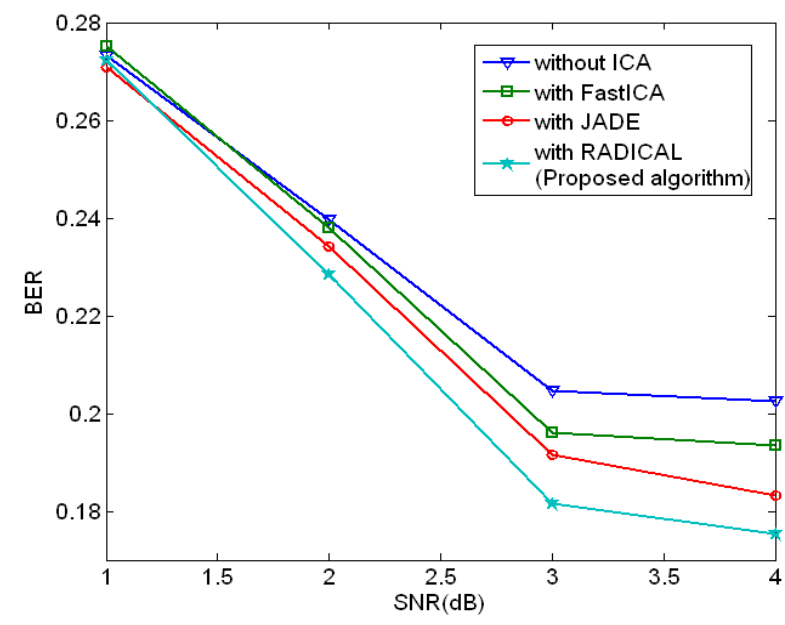

Fig 6: $S N R$ vs BER curves for $k=10$ in AWGN channel

\section{CONCLUSIONS}

Independent component analysis algorithm technique combined with conventional detection is used to improve BER performance in multi-user DS-CDMA environment. Based on the simulation results, It is observed that the BER performance of the proposed RADICAL ICA based multiuser DS-CDMA detection is better when compared with Fast ICA and JADE ICA based detection. In this paper the report on the observation made to justify the above fact is displayed. It is also identified that the performance using RADICAL 
ICA is more significant when compared with other ICA techniques mentioned above in the high signal to noise ratio scenario. In the proposed technique, the performance of ICA algorithm for detection is constrained by SNR and number of users of system. In the scenario of high SNR and low number of users ICA performs well for detection of DS-CDMA signals.

\section{REFERENCES}

[1] U. Madhow, "MMSE interference suppression for Direct Sequence Spread Spectrum CDMA", IEEE Transactions on communication, vol 42, no 12, pp 3178-3188, Dec 1994.

[2] S. Verdu. Multiuser Detection, Cambridge University Press, 1998.

[3] P. Comon. "Independent component analysis - a new concept". Signal Processing, 36:287-314, 1994.

[4] M. Honig, U. Madhow and S. Verdu, "Blind adaptive multi user detection", IEEE Transaction on Information Theory, vol 41, pp 944-960, July 1995.

[5] T. Ristaniemi, J. Joutsensalo, "Independent component analysis with code information utilization in DS-CDMA signals separation", Proceedings of the IEEE Global Telecommunications Conference, Brazil, 1999, pp. 320324.

[6] T. Ristaniemi. and J. Joutsensalo, "Advanced ICA Based Receivers for DS-CDMA systems", PIMRC, pp.276 $281,2000$.
[7] W.Y. LEONG, J. Homer, "Implementing ICA in Blind Multiuser Detection", International Symposium on Communications and Information Technologies 2004 (ISCIT 2004) Sapporo, Japan, October 26- 29, 2004.

[8] Jean-François Cardoso, "High-order contrasts for independent component analysis", Neural Computation, vol. 11, no 1, Jan. 1999, pp. 157-192.

[9] Erik G. Miller and John W. Fisher III, "Independent components analysis by direct entropy minimization," Tech. Rep. UCB/CSD-03-1221, University of California at Berkeley, January 2003

[10] Erik G. Learned-Miller, John W. Fisher III, "ICA Using Spacings Estimates of Entropy" Journal of Machine Learning Research 4 (2003) 1271-1295; Submitted 10/02; Published 12/03.

[11] Oldrich Vasicek, "A test for normality based on sample entropy," Journal of the Royal Statistical Society, Series B, vol. 38, no. 1, pp. 54-59, 1976

[12] A. Hyvärinen and E. Oja, "A fast fixed-point algorithm for independent component analysis," Neural Computation, vol. 9, pp.1483-1492, 1997.

[13] A. Hyvärinen. "Fast and robust fixed-point algorithms for independent component analysis", IEEE Transactions on Neural Networks, 10(3):626-634, May 1999.

[14] A. Hyvärinen (1997a), "A family of fixed-point algorithms for independent component analysis", In Proc. IEEE Int. Conf. on Acoustics, Speech and Signal Processing (ICASSP'97), pp 3917-3920, Munich, Germany, April 1997.

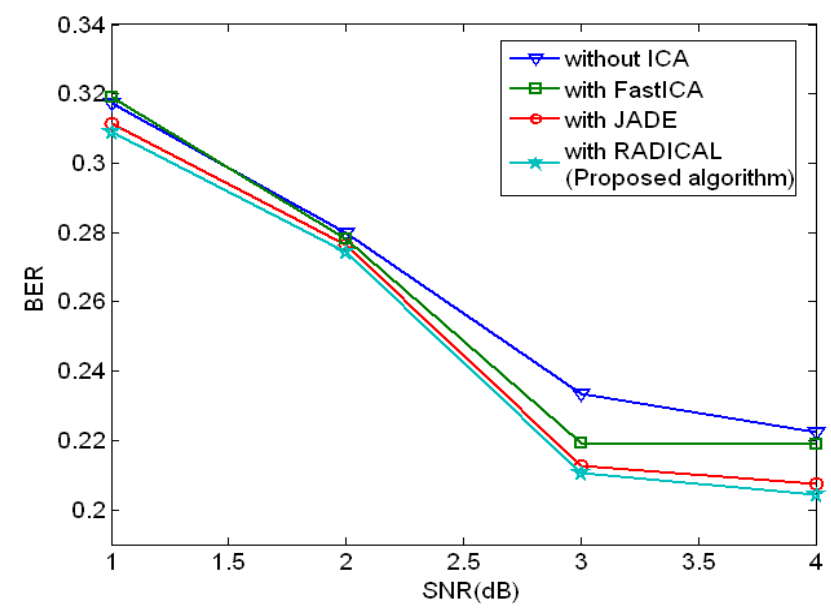

Fig 7: SNR vs BER curves for $k=20$ in AWGN channel 